\title{
Implementation of distress screening in an oncology setting
}

\author{
Timothy Pearman, PhD, ABPP, acb Sofia Garcia, PhD, abc Frank Penedo, PhD, ${ }^{\text {ac }}$ Betina Yanez \\ $\mathrm{PhD},{ }^{\text {ac }}$ Lynne Wagner, $\mathrm{PhD},{ }^{\mathrm{d}}$ and David Cella, $\mathrm{PhD}{ }^{\text {ac }}$
}

Departments of ${ }^{a}$ Medical Social Sciences and ${ }^{b}$ Psychiatry \& Behavioral Sciences, Northwestern University Feinberg School of Medicine, Chicago, Illionois; 'Robert H Lurie Comprehensive Cancer Center of Northwestern University, Chicago, Illinois; and ${ }^{\mathrm{d}}$ Department of Social Sciences and Health Policy, Wake Forest Medical School, Winston-Salem, North Carolina

The recommendations of numerous groups, such as the Institute of Medicine and the National Comprehensive Cancer Network, have resulted in the first regulatory standard on distress screening in oncology implemented in 2015 by the American College of Surgeons Commission on Cancer. This practice-changing standard promises to result in better quality cancer care, but presents unique challenges to many centers struggling to provide high-quality practical assessment and management of distress. The current paper reviews the history behind the CoC standard, identifies the most prevalent symptoms underlying distress, and discusses the importance of distress screening. We also review some commonly used instruments for assessing distress, and address barriers to implementation of screening and management.

\section{$\mathrm{T}$} The American College of Surgeons Commission on Cancer $(\mathrm{CoC})$ was established in 1913 with the goal of improving cancer care delivery. To date, more than 1,500 cancer centers are accredited nationwide. This means that about $70 \%$ of newly diagnosed cancer patients are treated at a CoC-accredited institution. ${ }^{1}$ In 2011, the $\mathrm{CoC}$ approved several new standards geared toward improving patient-centered continuum of care, including survivorship care plans, patient navigation, and distress screening. ${ }^{2}$ Cancer committees at all $\mathrm{CoC}$-accredited cancer centers are expected to develop a plan to screen for distress and to provide for meaningful treatment or referral for addressing this distress when it is identified in a given patient.

The standard builds on the work of the Institute of Medicine (IOM) which, in 2007, produced a landmark report, Cancer Care for the Whole Patient. ${ }^{3}$ The report suggested that despite remarkable advances in cancer treatment over the previous 30 years, the management of the psychosocial sequelae of cancer had not kept pace. The report further suggested that adequate psychosocial care needed to be considered standard care for all patients. The IOM report was followed by a recommendation by the distress management panel of the National Comprehensive Cancer Network (NCCN) that distress screening and management needed to be provided to all patients, regardless of diagnosis or stage of disease. ${ }^{4}$
This paper reviews the existing literature in several relevant areas. First, we review symptom prevalence in cancer and its impact on patient quality of life. Second, we discuss the impact of distress screening on patient outcomes. Third, we identify challenges in assessing symptoms such as depression from both provider and health care system levels. Fourth, we review methods of screening and specific instruments, highlighting the importance of using practical and validated patient self-report measures. Finally, we discuss barriers and implementation strategies for academic and community practice.

\section{Symptom prevalence and impact of distress}

Cella and colleagues highlighted the prevalence of the most distressing symptoms in oncology settings. ${ }^{5}$ The list, in order of frequency (highest to lowest), includes fatigue, pain, anxiety, sadness, and nausea. Understandably, clinically significant psychological distress is approximately twice as prevalent in cancer patients compared with the general population. Consequences of this elevated distress are critical impairments in general well-being and quality of life. ${ }^{6}$ In some studies, elevated distress has also been linked to poorer health outcomes, such as higher mortality, greater morbidity, and poorer immune functioning. ${ }^{7,8}$ In addition, other outcomes such as medical expenditures and occupational func-

Accepted for publication November 5, 2015. Correspondence: Timothy Pearman, PhD, ABPP; t-pearman@northwestern. edu. Disclosures: The authors have no disclosures. JCSO 2015;13:423-428. (2015 Frontline Medical Communications. DOI 10.12788/jicso.0198. 
TABLE Advantages and disadvantages of distress screening methodologies

\section{Clinician administered}

- Allow immediate assessment in clinic

- Allow for immediate referral in clinic

- Can be administered to patients who cannot complete self-assessments (eg, low literacy, certain visual or motor limitations)

- Clinician time burden

- Vulnerable to clinician bias. Clinicians must be properly trained or validity will be in question

Disadvantages Vulnerable to patient response bias such that they may be less likely to disclose distress if clinician(s) present.

- Non-mental health professionals may not know how to respond to elevated distress.

\section{Patient administered}

- Allow greater patient privacy

- Can be administered electronically in clinic or from patients' homes

- Likely to get more 'honest' patient responses

- Necessitates that a system be put in place to ensure results are dealt with in a reasonable time frame

- Barriers with low literacy, non-English language speakers, poor computer skills

- Family members may complete assessment, rendering it invalid tioning may be adversely affected in patients experiencing serious psychological distress. ${ }^{9,10}$

The most common and straightforward approach to measure distress is though the clinician's impression of emotional state during a clinic visit. However, we know that such approaches often result in health care providers doing a relatively poor job of estimating patient distress. ${ }^{11,12,13}$ In particular, health care professionals tend to underestimate the degree of patients' distress. Further, assessment of depression seems to be the most underestimated among common symptoms. ${ }^{14}$ This is concerning in light of the negative outcomes in patients with serious psychological distress. It also highlights the importance of using validated patient-reported outcomes (PRO) measures, rather than proxy measures (ie, estimates of patient distress given by family members or health care providers).

\section{Methods and management of distress screening}

$\mathrm{PRO}$ instruments are the accepted standard for accurate estimation of distress. There are many PRO instruments available for use. These have been described elsewhere: a joint task force of the American Psychosocial Oncology Society (APOS), the Association of Oncology Social Work (AOSW) and the Oncology Nursing Society (ONS) has provided an excellent summary of PRO instruments; ${ }^{15}$ and other authors have also addressed the topic. ${ }^{16,17}$

Before choosing a PRO instrument, it is important to ensure that the treatment setting possesses someone with the requisite skills to interpret and manage the distress screener. $\mathrm{CoC}$ guidelines are vague on what constitutes qualification for managing distress; generally, a health care provider trained in management of emotional distress will qualify. For centers in which there are no mental health professionals available, there are a number of training courses available for physicians, nurses, and allied health professionals. These include a free, online course offered by APOS (www.apos-society.org), and courses offered by the ONS (http://www2.ons.org/CourseDetail.aspx?course_ $\mathrm{id}=87$ ) and the AOSW (www.aosw.org).

There are 2 approaches to screening: clinician-based and patient-based administration. Both have advantages and disadvantages (Table). Clinician-administered screenings have the benefit of allowing clinicians to assess distress immediately, in clinic, and then act on those screening results with treatment or referral. However, this technique is time intensive and can be vulnerable to reporting bias, in that patients may be more likely to honestly disclose their distress if clinicians were not present. In addition, error may be introduced if clinicians are not properly trained in how to administer these instruments (eg, asking leading questions may lead to under- or over-reporting). Finally, non-mental health professionals may not know how to adequately respond to elevated levels of distress.

Patient-administered screening tools can consist of either pencil-and-paper instruments, or electronic distress screeners. There are now a number of electronic distress screening tools available..$^{18,19,20}$ In general, these tools are intended to seamlessly integrate with existing electronic medical records (EMRs), and patients can complete the screenings at home through a patient portal or in clinic on a tablet, laptop, or smartphone devices. Some of these instruments provide patients with referral information and educational materials. They are also designed to automatically place the screening information in the patient's EMR. These instruments allow greater privacy but necessitate that a system be put in place so that the screenings are evaluated within a reasonable frame and a response is enacted for high levels of distress. In addition, there can be barriers for patients with low literacy, poor computer skills, or who do not speak English. It is also possible that family members might complete the questionnaire on behalf of the patient, which renders patient-reported measures invalid.

The most widely used patient-administered instrument is the NCCN Distress Thermometer (DT) and Problem List. ${ }^{21}$ That approach has patients rate their distress on a 
scale of 0-10 (0, least distress), and then accompanies the rating with a problem list of about 40 items asking patients to specify any problems that are contributing to their distress. The tool has high face validity, is easily understandable, and has generally acceptable psychometric properties. It has been validated across multiple languages, adding to its utility. ${ }^{22}$ However, despite its practicality, it performs poorly in assessing depression because of its low specificity and sensitivity, and therefore we recommend pairing it with a depression-specific instrument, such as the PHQ-2 (Patient Health Questionnaire-2). ${ }^{23}$ A recently developed tool is the brief 7-item short version of the widely used FACT-G (Functional Assessment of Cancer Therapy: General), referred to as the FACT-G7. This is a brief, wellvalidated instrument measuring quality of life in cancer patients. ${ }^{24}$ One of the 7 items taps the anxiety component of distress, with clinically significant cut scores, and provides a good group-level estimate of FACT-G score.

\section{The distress management process in action}

After choosing an appropriate tool, the next step is to design a process by which patients' distress screening responses are evaluated and an appropriate response is initiated when warranted. Important considerations include designating who will be responsible for administering, collecting, and reviewing the data. This can be the same person or multiple individuals, but accountability is key to ensure that high levels of distress do not go unnoticed and that responses to distress are timely. For patients with high levels of distress (ie, generally a score of 4 or higher on the NCCN-DT), a clinician or team of clinicians should review the assessment and follow-up with referral or treatment. The NCCN distress management guidelines should be available to guide referrals and treatment after identification of significant distress.

At the Robert H Lurie Comprehensive Cancer Center of Northwestern University we have been administering the Patient-Reported Measurement Information System (PROMIS) anxiety and depression computer-adaptive tests (CATs) since December 2014. PROMIS CATs use a computer algorithm developed with item response theory to administer PRO items, selected from an item bank, which are tailored to each patient's symptom severity (for more detail, see Wagner and colleagues ${ }^{25}$ ). An item bank comprises items representative of the spectrum of a common trait (eg, depression) that are calibrated on the same measurement scale, thus simplifying scoring and interpretation (including T-score cut-offs for identifying elevated scores). PROMIS T-scores range from 1-100, with 50 representing the national average and 10 points being 1 standard deviation. Higher scores indicate more of the construct so, for example, a higher score for depression indicates more depression. At our institution, PROMIS

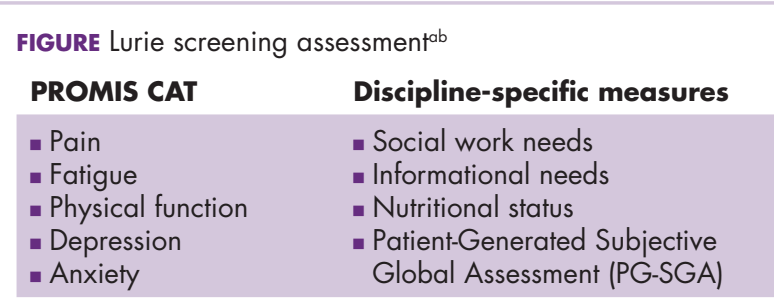

PROMIS CAT, Patient-Reported Measurement Information System ComputerAdaptive Tests

${ }^{\circ} 40$ items, takes less than 10 minutes. ${ }^{b}$ Wagner et al. ${ }^{25}$

CAT administration, scoring, and real-time clinician notification of elevated scores are integrated within the EMR (EPIC).

The CATs used at our institution assesses 5 primary domains: fatigue, pain, physical functioning, depression, and anxiety. Those domains were chosen because they represent the most commonly reported symptoms and concerns within the oncology setting. In addition to those domains, patients are also given the opportunity to request a response from a social worker for practical problems such as transportation, insurance, child care, and so on. They may also ask for more information about their diagnosis, treatment, and so on, and their requests are routed to our oncology health education coordinator. Finally, patients may request a response from a dietitian for any nutritional concerns. The assessment length is approximately $40 \mathrm{items}$ and on average takes slightly less than 10 minutes to complete (Figure).

Patients receive a notification through the electronic health record patient-facing portal (MyChart) 72 hours before any scheduled appointment with their medical oncologist (the system has not yet been extended to radiation oncology and surgical oncology). Patients are asked to click on a link, which takes them directly to the assessment. At the end of the assessment they are informed that it may take up to 72 hours for the results to be evaluated by the health care team and if they are experiencing any emergencies, they should call 911 or proceed immediately to the nearest emergency department.

Assessment results are immediately populated in the EMR in 3 ways. First, all assessment results, including the date completed, PROMIS T-scores, severity interpretation (normal, mild, moderate, or severe), items administered, and patient responses are documented in the EMR. Second, providers can copy assessment results into progress notes in the EMR. Third, clinicians are alerted to severe symptoms and patient concerns through messages sent to their EMR in-basket. Patients' PROMIS T-scores in the severe range ( $\geq 70$ or 75 , depending on the symptom) trigger electronic provider notification through medical record messaging to the primary oncologist and a nurse messag- 
ing pool. Severe scores on depression and anxiety are also routed to our social work provider pool for triaging.

When designing our electronic system, it is imperative that all distress screenings should be documented in patients' medical records. With electronic screening systems, results are typically automatically populated in the EMR. Otherwise, clinicians will be responsible for documenting the result, and most importantly, the response to the distress screening result. Thus, automatic documentation of results is important to promote both appropriate clinical follow-up and program evaluation (ie, determining whether the system is working properly or modifications are necessary).

\section{Distress screening and improved outcomes}

Because distress screening has only recently become mandatory in $\mathrm{CoC}$-accredited cancer centers, the literature is fairly sparse in this regard. However, a number of welldesigned studies have found that if a systematic approach is taken to distress screening, evaluation, and referral, it is indeed effective. Specifically, research indicates that clinician documentation is improved, as is referral for distress. ${ }^{26}$ Further, patient distress is minimized, patient educational needs are decreased, and communication with physicians and health care providers increase. ${ }^{27,28}$ It should be noted, however, that the available evidence suggests that screening in the absence of an established triage plan does not improve outcomes. ${ }^{28}$

\section{Barriers to screening}

Less than 2 years ago, a survey of $70 \mathrm{CoC}$-accredited institutions found that fewer than half of them (29) had begun distress screening. ${ }^{30}$ Barriers in this study were identified by self-report from institutions. The primary barriers were lack of information on how to implement the recommended screening; lack of buy-in among key cancer center staff, including oncologists; and lack of information on how to pilot test screening before expanding center wide. In addition to those barriers to beginning a distress screening protocol, another significant barrier was ensuring that appropriate referrals are made when elevated distress is found in a given patient.

Another recent study found that rates of distress screening compliance varied across different clinics, ranging from $47 \%-73 \%$ of eligible patients being screened at least once during their treatment. ${ }^{31}$ Of note, however, is that it seemed that time since beginning a screening protocol was positively correlated with screening rates. That suggests that clinics may improve at distress screening over time, perhaps as a result of quality improvement processes. However, it should also be noted that this study looked at academic medical centers, which would be expected to have higher rates of screening than would a similar sample of commu- nity oncology practices. This is important because there is very limited information about the prevalence and effectiveness of distress screening in community-based hospitals and clinics. And it is worth noting that a disproportionate number of minorities and vulnerable populations are served in such community hospitals and clinics.

Another practical barrier that we encountered at our center revolved around questions we received from nurses and medical assistants about how to perform clinician-administered screening. After beginning NCCN-DT clinicianadministered screening several years ago, we met with our nurses and medical assistants to assess their experiences with screening. They indicated candidly that they were not performing the screening correctly, but were merely "guessing" at the person's level of distress when they met them in clinic. When asked why this was the case, they mentioned 3 barriers to appropriate screening: lack of training on how to administer the NCCN-DT, lack of competence in how to refer patients when significant distress was identified, and discomfort with the amount of time they perceived it would require if a patient presented with elevated distress and they then had to spend additional time addressing those concerns. The clinicians were worried that their clinic flow would be interrupted, leading to long delays and dissatisfied patients and physicians.

This feedback led to several follow-up meetings to train staff on protocols for distress screening and to provide them with skills to appropriately implement screening and referral. We eventually decided to implement electronic distress screening in our clinic so that the problems would be overcome systemically and there would be no barriers to the smooth, operational flow of patients through our clinic. ${ }^{25}$ This is a good example that highlights the importance of obtaining feedback from the stakeholders in distress screening and how clinics should tailor screening methods to their individual practice patterns.

\section{Summary and recommendations}

The first regulatory standard on distress screening, mandated by the $\mathrm{CoC}$ went into effect in January 2015. This practice-changing standard promises to improve the psychosocial care of oncology patients by identifying areas leading to significant distress and providing resources for referring patients for appropriate treatment. It has been known for some time that depression, anxiety, fatigue, and other distressing symptoms are significantly elevated in cancer patients compared with the general population. These distressing symptoms can lead to poorer outcomes that include not just impaired quality of life, but increased morbidity and mortality as well. Unfortunately, oncology providers have difficulty identifying significant distress in their patients. It is precisely this difficulty that has led to research over the last 20 years investigating measurement of PROs.

$\mathrm{PRO}$ tools remain the best way of assessing distress 
in oncology patients. Screening tools that use PROMIS measures and tools such as the FACT-G7 have been well validated and are user friendly. The choice of a method of administration, as well as the specific screening tool, must be based on an assessment of clinic volume, needs, and the staff available to administer the screening and assist with referrals for patients exhibiting elevated distress. Several recent articles highlight these issues as well as others, such as cultural relevance. ${ }^{32,33}$ There are a number of online courses, some free, which are available to train professionals to administer screening.

Distress screening, performed appropriately, has been shown to positively impact outcomes such as quality of life. ${ }^{27}$ However, it is incumbent on the health care system to ensure that quality monitoring is performed, such that performance improvement measures can be taken to address barriers or operational difficulties. It should be noted that in any cancer center - from small community practices to large academic practices - barriers will exist and should be expected. It is promising that time since implementation of distress screening is positively correlated with rates of administration, such that more experienced centers have higher rates of screening. When it comes to distress screening, practice may make perfect (or at least much improved).

Several needs for future research are apparent. First, studies should investigate the efficacy, ease of administration, and PROs associated with various distress screening methodologies (eg, clinician-administered vs patientreported). Second, the impact on patient and system outcomes of single screening (as mandated by the $\mathrm{CoC}$ standard) compared with repeated screenings (such as those performed with many of the electronic screenings) is unknown. In addition, the most successful distress screening programs seem to incorporate staff training. Therefore, studies are needed to evaluate the most effective means of training staff and the effect of training on implementation and outcomes. Third, because distress screening brings associated costs, future research should investigate the most cost-effective means of implementing screening, as well as measuring any potential and actual cost savings to the health care system based on screening (eg, reduced inpatient admissions, emergency department visits, and so on) Future research would do well to enhance the value of what is already a practice-driving change in the standard of cancer care delivered in the United States.

\section{Acknowledgments}

This work was funded by support from the Robert $\mathrm{H}$ Lurie Comprehensive Cancer Center and the Coleman Foundation.

\section{References}

1. Wagner LW, Pearman TP. Changes in US policy: psychosocial care as an integral component of cancer care delivery. In: Holland et al (eds), Psycho-Oncology (3 ed.), chapter 103. Springer: New York; February 2015.
2. American College of Surgeons. Cancer Program Standards 2012 Version 1.2.1: Ensuring Patient-Centered Care. http://www.facs.org/ cancer/coc/cocprogramstandards2012.pdf. Accessed August 2014.

3. Adler NE, Page AEK. Cancer care for the whole patient: meeting psychosocial health needs. Institute of Medicine (US) Committee on Psychosocial Services to Cancer Patients/Families in a Community Setting. National Academies Press. 2008.

4. Holland JC. Distress screening and the integration of psychosocial care into routine oncologic care. J Natl Compr Canc Netw. 2013;11(5 suppl): 687-689.

5. Cella D, Peterman A, Passik S, Jacobsen P, Breitbart W. Progress toward guidelines for the management of fatigue. Oncology. 2008;12:369-377.

6. Pearman TP, Yanez B, Peipert JD, Wortman K, Beaumont J, Cella D. Ambulatory cancer and US general population reference values and cutoff scores for the Functional Assessment of Cancer Therapy. Cancer. 2014;120:2902-2909.

7. Rodrigue JR, Pearman T, Moreb J. Morbidity and mortality following bone marrow transplantation: predictive utility of pre-BMT affective functioning, compliance, and social support stability. Int J Behav Med. 1999;6:241-254.

8. Barrera I, Spiegel D. Review of psychotherapeutic interventions on depression in cancer patients and their impact on disease progression. Int Rev Psychiatry. 2014;26:31-43.

9. Han X, Lin CC, Li C, et al. Association between serious psychological distress and health care use and expenditures by cancer history. Cancer. 2015;121:614-622.

10. Manne SL, Siegel S, Heckman CL, Kashy DA. Psychological distress as a mediator of the association between disease severity and occupational functioning among employed spouses of women recently diagnosed with breast cancer. Psychooncology. 2015;24:1560-1568.

11. Fallowfield L, Ratcliffe D, Jenkins V, Saul J. Psychiatric morbidity and its recognition by doctors in patients with cancer. $\mathrm{Br} \mathrm{J} \mathrm{Cancer.}$ 2001;84:1011-1015.

12. Keller M, Sommerfeldt S, Fischer C, et al. Recognition of distress and psychiatric morbidity in cancer patients: a multi-method approach. Ann Oncol. 2004;15:1243-1249.

13. Merckaert I, Libert Y, Delvaux N, et al. Factors that influence physicians' detection of distress in patients with cancer: can a communication skills training program improve physicians' detection? Cancer. 2005;104: 411-421.

14. Gouveia L, Lelorain S, Bredart A, et al. Oncologists' perception of depressive symptoms in patients with advanced cancer: accuracy and relational correlates. BMC Psychol. 2015;3:6.

15. Pirl WF, Fann JR, Greer JA, et al. Recommendations for the implementation of distress screening programs in cancer centers. Cancer. 2014;120:2946-2954.

16. Petty L, Lester J. Distress screening in chronic disease: essential for cancer survivors. J Adv Pract Oncol. 2014;5:107-114.

17. Grassi L, Caruso R, Sabato S, et al. Psychosocial screening and assessment in oncology and palliative care settings. Front Psychol. 2015;5:1485.

18. Abernethy AP Ahmad A, Zafar SY, et al. Electronic patient-reported data capture as a foundation of rapid learning cancer care. Medical Care. 2010;48(6 suppl): S32-38.

19. Clark K, Bardwell WA, Arsenault T, DeTeresa R, Loscalzo M. Implementing touch-screen technology to enhance recognition of distress. Psychooncology. 2009;18:822-830.

20. Miller MF, Buzaglo JS, Clark KL, et al. Demonstrating the psychometric properties of a problem-related distress screener in a community sample of 319 cancer survivors. Psychooncology. 2013;22:1249-1257.

21. Jacobsen PB, Donovan KA, Trask PC, et al. Screening for psychologic distress in ambulatory cancer patients. Cancer. 2005;103:14941502.

22. Donovan KA, Grassi L, McGinty HL, Jacobsen PB. Validation of the distress thermometer worldwide: state of the science. Psychooncology. 2014;23:241-250.

23. Wagner LW, Spiegel D, Pearman T. The American College of Surgeons Commission on Cancer's new screening standards for integration of psychosocial care/distress management into routine care. J 


\section{How We Do It}

Natl Compr Canc Netw. 2013;11:1-8.

24. Yanez B, Pearman T, Beaumont JL, Lis CG, Cella, D. The FACTG7: a rapid version of the Functional Assessment of Cancer Therapy General (FACT-G) for monitoring symptoms and concerns in oncology practice and research. Ann Oncol. 2013;24:1073-1078.

25. Wagner, LI, Schink, J, Bass, M, et al. Bringing PROMIS to practice: brief and precise symptom screening in ambulatory cancer care. Cancer. 2015;33:927-934.

26. Chiang AC, Buia Amport S, Corjulo D, Harvey KL, McCorkle R. Incorporating patient-reported outcomes to improve emotional distress screening and assessment in an ambulatory oncology clinic. J Oncol Pract. 2015;11:219-222.

27. Carlson LE, Groff SL, Maciejewski O, Bultz BD. Screening for distress in breast and lung cancer outpatients: a randomized, controlled clinical trial. J Clin Oncol. 2010;28:4884-4891.

28. Carlson LE, Waller A, Mitchell AJ. Screening for distress and unmet needs in patients with cancer: review and recommendations. J Clin Oncol. 2012;30:1160-1177.
29. Hollingworth W, Metcalfe C, Mancero S, et al. Are needs assessments cost effective in reducing distress among patients with cancer? A randomized controlled trial using the Distress Thermometer and Problem List. J Clin Oncol. 2013;31:3631-3638.

30. Lazenby M, Ercolano E, Grant M, Holland JC, Jacobsen PB, McCorkle R. Supporting commission on cancer mandated psychosocial distress screening with implementation strategies. J Oncol Pract. 2015;11:e413-420.

31. Zebrack B, Kayser K, Sundstrom L, et al. Psychosocial distress screening implementation in cancer care: an analysis of adherence, responsiveness and acceptability. J Clin Oncol. 2015;33:1165-1171.

32. Kayser K, Acquati C, Tran TV. No patient left behind: a systematic review of the cultural equivalence of distress screening instruments. J Psychosoc Oncol. 2012;30:679-693.

33. Rohan EA. Removing the stress from selecting instruments: arming social workers to take leadership in routine distress screening implementation. J Psychosoc Oncol. 2012;30:667-678. 